\title{
Exacerbations of severe asthma in patients treated with mepolizumab
}

\author{
To the Editor:
}

Mepolizumab, a humanised monoclonal antibody that neutralises interleukin-5, reduces exacerbations of severe eosinophilic asthma and chronic obstructive pulmonary disease (COPD) [1,2]. The beneficial effect of treatment is most obvious in patients with a raised peripheral blood eosinophil count, a group who are at high risk of exacerbation off treatment $[2,3]$. Even in this population, exacerbation rates whilst receiving mepolizumab are around one per patient per year. The nature of these remaining exacerbations has not been described. We carried out a post hoc comparison of exacerbations occurring during treatment with mepolizumab or placebo in a previously reported, double-blind, placebo-controlled trial of mepolizumab in severe eosinophilic asthma [1]. We tested the hypothesis that exacerbations in each group differ with respect to change in symptom scores, forced expiratory volume in $1 \mathrm{~s}(\mathrm{FEV} 1)$ and inflammatory profile.

We carried out a retrospective analysis of exacerbations occurring during a placebo-controlled, double-blind trial of mepolizumab $750 \mathrm{mg}$ i.v. given every 4 weeks for 52 weeks [1]. The study involved 61 patients with severe asthma, who had an induced sputum eosinophil count of $>3 \%$ at screening or at some point over the previous 12 months, and two or more exacerbations of asthma treated with systemic corticosteroids in the last year. 29 patients were treated with mepolizumab and 32 with placebo. Patients were asked to contact the study team for assessment at the time of a clinical deterioration suggestive of an exacerbation, ideally prior to starting rescue treatment according to their self-management plan. Patients were seen prior to starting rescue prednisolone treatment or as soon as possible after. Cough, breathlessness and wheeze visual analogue scale (VAS) [4], spirometry, Asthma Control Questionnaire (ACQ5) and induced sputum inflammatory cell counts were measured. Exacerbations were analysed as individual events. Further analysis was conducted at the patient level to investigate whether there was a significant effect of clustering due to some patients having multiple exacerbations.

Exacerbation data were available for 159 episodes treated with oral prednisolone. 105 events occurred in 25 patients in the placebo arm and 54 events in 18 patients in the mepolizumab arm. In 83 exacerbations (28 on mepolizumab and 55 on placebo) patients were reviewed prior to prednisolone treatment and in 76 (26 mepolizumab and 50 placebo) patients were seen after starting prednisolone for a mean \pm SD of $5.4 \pm 8.8$ days in the placebo arm and $5 \pm 7.5$ days in the mepolizumab arm. The median number of exacerbations per patient was three (range 1-9).

Overall, exacerbations on placebo were associated with a higher sputum eosinophil percentage than those on mepolizumab (geometric mean $5.4 \%$ versus $2.6 \%, \mathrm{p}=0.03$ ). There was no difference in total VAS, ACQ5, FEV1 or change in these measures from the baseline study visit or immediately pre-exacerbation visit between the placebo and mepolizumab groups.

However, closer examination of the data showed that there were marked differences in the patients seen before and those seen during rescue prednisolone treatment. In patients seen prior to rescue prednisolone treatment, change from baseline in symptom VAS was greater (40versus $16 \mathrm{~mm}$; mean difference $24 \mathrm{~mm}$, 95\% CI 15-34 mm; p<0.001) and geometric mean sputum eosinophil count was higher (6.7 versus 2.6\%; $\mathrm{p}=0.046)$ in the placebo arm than the mepolizumab arm. In contrast, in patients seen when taking rescue prednisolone treatment, patients on placebo tended to have a lower mean change in VAS than patients on mepolizumab (14 versus $28 \mathrm{~mm}$; mean difference $-14 \mathrm{~mm}, 95 \%$ CI $-3-31 \mathrm{~mm}$; $=0.1$ ) (table 1 ) and a smaller difference in geometric mean sputum eosinophil count (2.7 versus $1.1 \%$; $=0.1$ ) (table 1). All

@ERSpublications

Exacerbations on mepolizumab are associated with a lower sputum eosinophil level appear less severe in terms of symptoms compared to placebo. These findings raise the possibility that an alternative treatment approach may be needed for these events. http://ow.ly/SoFX30md6of

Cite this article as: Shrimanker R, Pavord ID, Yancey S, et al. Exacerbations of severe asthma in patients treated with mepolizumab. Eur Respir J 2018; 52: 1801127 [https://doi.org/10.1183/13993003.01127-2018]. 
TABLE 1 Patient characteristics at exacerbation assessment

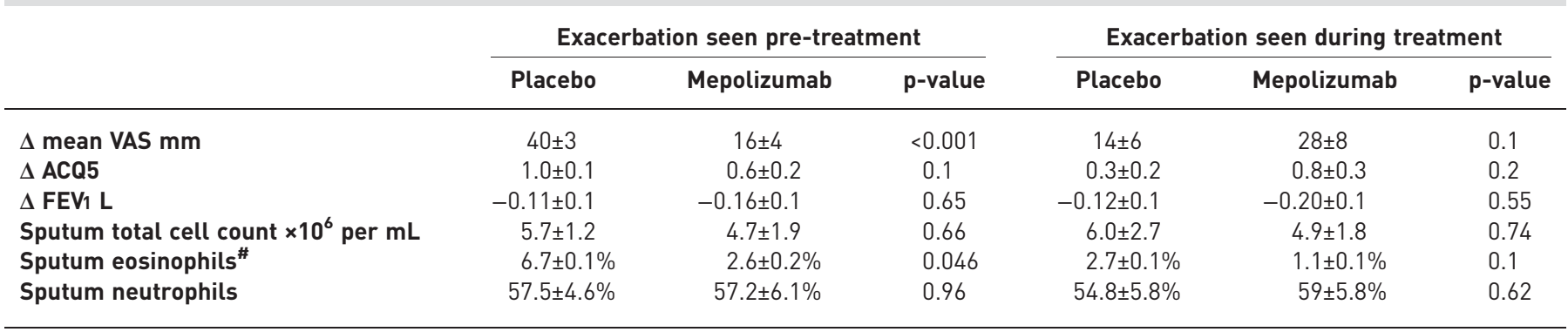

Data are presented as mean \pm SE unless otherwise stated. $\Delta$ : change from baseline; VAS: visual analogue scale; ACQ5: Asthma Control Questionnaire; FEV1: forced expiratory volume in $1 \mathrm{s.}{ }^{\#}$ : geometric mean \pm logsE.

elements of the total VAS (cough, wheeze and breathlessness) were equally affected. There were no differences in the sputum total cell count or mean sputum neutrophil differential count (table 1).

There was no significant difference in the change from baseline ACQ5 or FEV1 in the placebo versus mepolizumab arms either in the pre-treatment or treated exacerbation groups (table 1). Similar results were seen if the last stable visit measurements were used in place of the baseline values.

The results were similar when these analyses were run using either the mean of each patient's exacerbation data or using the data from their first exacerbation, suggesting that our findings were not driven by individual patients with frequent events.

The most striking finding of our retrospective analysis of exacerbations of severe eosinophilic asthma occurring whilst on mepolizumab and placebo is the marked difference in change in VAS in patients assessed before and after rescue prednisolone was started. In patients on placebo, the change in VAS from a stable state was much greater when assessed before, compared to after, rescue treatment with prednisolone for several days. This difference was not evident in patients receiving mepolizumab. The most plausible explanation for these observations is that exacerbation events occurring on mepolizumab are both less severe in terms of symptoms, and less responsive to prednisolone.

There is evidence to support this interpretation. Exacerbations occurring on mepolizumab were associated with a lower induced sputum eosinophil count compared to those occurring on placebo, particularly in the group who had not started rescue prednisone. Moreover, studies in asthma [5] and COPD [6] have shown that non-eosinophilic exacerbations are less severe, and are associated with a longer duration and a poorer response to corticosteroids than eosinophilic episodes [6-8].

We saw similar trends when symptoms were assessed by ACQ5, although this measure proved to be less responsive than total VAS, probably because it assesses symptoms over 1 week. We found no evidence of a differences in change in FEVI at exacerbation, suggesting that changes in this measure are disassociated from changes in symptom scores.

Our findings are preliminary and are based on a post hoc retrospective analysis, so some caution is required. However, they are potentially important as they suggest that different mechanisms might drive exacerbations occurring whilst on mepolizumab treatment. This raises the possibility that different, potentially non-corticosteroid based treatment approaches may be sufficient for those exacerbations that are not eosinophilic in nature. Future studies evaluating the symptom, physiological, airway inflammatory and microbiological characteristics of exacerbation events occurring on mepolizumab are required to investigate this possibility.

Rahul Shrimanker ${ }^{1}$, Ian D. Pavord ${ }^{1}$, Steve Yancey $^{2}$, Liam G. Heaney ${ }^{3}$, Ruth H. Green ${ }^{4}$, Peter Bradding ${ }^{4}$, Beverley Hargadon ${ }^{4}$, Chris E. Brightling ${ }^{4}$, Andrew J. Wardlaw ${ }^{4}$ and Pranabashis Haldar ${ }^{4}$

${ }^{1}$ Respiratory Medicine Unit, Nuffield Dept of Medicine and Oxford Respiratory BRC, University of Oxford, Oxford, UK. ${ }^{2}$ Respiratory Medical Franchise and the Respiratory Therapeutic Area, GlaxoSmithKline, Research Triangle Park, NC, USA. ${ }^{3}$ Dept of Respiratory Medicine, Centre for Experimental Medicine, School of Medicine, Dentistry and Biomedical Sciences, Queen's University Belfast, Belfast, UK. ${ }^{4}$ Dept of Respiratory Medicine, Allergy and Thoracic Surgery and NIHR BRC Respiratory Theme, Glenfield Hospital, University of Leicester, Leicester, UK.

Correspondence: Ian D. Pavord, Respiratory Medicine Unit, Nuffield Department of Medicine and Oxford Respiratory BRC, University of Oxford, NDM Research Building, Old Road Campus, Oxford OX3 7FZ, UK.

E-mail: ian.pavord@ndm.ox.ac.uk 
Received: June 222018 | Accepted after revision: Oct 042018

This study is registered at www.isrctn.com with identifier number ISRCTN75169762. Individual participant data will not be made available.

Author contributions: R. Shrimanker, I.D. Pavord, S. Yancey, P. Haldar and L.G. Heaney conceived and designed the study; R. Shrimanker, R.H. Green, P. Haldar, B. Hargadon, C.E. Brightling, A. J. Wardlaw, P. Haldar and I.D. Pavord acquired, analysed and interpreted the data; and R. Shrimanker, I.D. Pavord and P. Haldar drafted the manuscript.

Conflict of interest: R. Shrimanker has nothing to disclose. I.D. Pavord reports receiving speaker's honoraria, travel expenses and honoraria for attending advisory boards from AstraZeneca, GSK, Boehringer Ingelheim and Teva, a research grant, speaker's honoraria, travel expenses and honoraria for attending advisory boards from Chiesi, honoraria for attending advisory boards from Sanofi/Regeneron, speaker's honoraria from Circassia and Mundipharma, honoraria for attending advisory boards from Merck, Novartis, Knopp and Roche/Genentech, a research grant and honoraria for attending advisory boards from Afferent, outside the submitted work. S. Yancey is an employee of GSK and holds stock in that company. L.G. Heaney reports reports that Vitalograph Ltd provided INCA inhaler technology as part of the MRC-funded RASP-UK programme and Aerocrine $\mathrm{AB}$ provided FeNO machines at reduced cost as part of that programme. He took part in advisory boards and gave lectures at meetings supported by GSK, Respivert, MSD, Nycomed, Boehringer Ingelheim, Teva, Vectura, Novartis and AstraZeneca, during the conduct of the study. He received sponsorship for attending international scientific meetings from AstraZeneca, Boehringer Ingelheim, GSK and Napp Pharmaceuticals, and received lecture and advisory board fees from Novartis, Roche Genentech Inc., Sanofi, GSK, Amgen, AstraZeneca and Aerocrine, outside the submitted work. R.H. Green reports receiving educational speaker fees from AstraZeneca and Novartis, outside the submitted work. P. Bradding reports receiving non-financial support (provision of the study drug) from GSK during the conduct of the study. B. Hargadon has nothing to disclose. C.E. Brightling reports receiving grants from GSK and the NIHR, during the conduct of the study; grants and personal fees from AZ/MedImmune, grants and personal fees from GSK, Boehringer Ingelheim, Chiesi, Novartis and Roche/Genentech, personal fees from Glenmark, Gilead and Teva, Sanofi, Regeneron, 4DPharma, PreP and Mologic, paid to his institution, outside the submitted work. A.J. Wardlaw reports receiving grants from GSK during the conduct of the study, and grants from Pulmocide, and personal fees from GSK, AstraZeneca/MedImmune, Teva and GfK, outside the submitted work. P. Haldar has nothing to disclose.

Support statement: This research was supported by the National Institute for Health Research (NIHR) Oxford Biomedical Research Centre (BRC) and the Leicester NIHR BRC. The views expressed are those of the author(s) and not necessarily those of the NHS, the NIHR or the Department of Health.

\section{References}

1 Haldar P, Brightling CE, Hargadon B, et al. Mepolizumab and exacerbations of refractory eosinophilic asthma. N Engl J Med 2009; 360: 973-984.

2 Pavord ID, Korn S, Howarth P, et al. Mepolizumab for severe eosinophilic asthma (DREAM): a multicentre, double-blind, placebo-controlled trial. Lancet 2012; 380: 651-659.

3 Ortega HG, Yancey SW, Mayer B, et al. Severe eosinophilic asthma treated with mepolizumab stratified by baseline eosinophil thresholds: a secondary analysis of the DREAM and MENSA studies. Lancet Respir Med 2016; 4: 549-556.

4 Green RH, Brightling CE, McKenna S, et al. Asthma exacerbations and sputum eosinophil counts: a randomised controlled trial. Lancet 2002; 360: 1715-1721.

5 Jayaram L, Pizzichini MM, Cook RJ, et al. Determining asthma treatment by monitoring sputum cell counts: effect on exacerbations. Eur Respir J 2006; 27: 483-494.

6 Bafadhel M, McKenna S, Terry S, et al. Blood eosinophils to direct corticosteroid treatment of exacerbations of chronic obstructive pulmonary disease: a randomized placebo-controlled trial. Am J Respir Crit Care Med 2012; 186: $48-55$.

7 Green RH, Brightling CE, McKenna S, et al. Asthma exacerbations and sputum eosinophil counts: a randomised controlled trial. Lancet 2002; 360: 1715-1721.

8 Green RH, Brightling CE, Woltmann G, et al. Analysis of induced sputum in adults with asthma: identification of subgroup with isolated sputum neutrophilia and poor response to inhaled corticosteroids. Thorax 2002; 57: $875-879$. 